\title{
Timing Calibration in PET Using a Time Alignment Probe
}

\author{
W. W. Moses, Fellow, IEEE, and C. J. Thompson, Member, IEEE
}

\begin{abstract}
We evaluate the Scanwell Time Alignment Probe for performing the timing calibration for the LBNL ProstateSpecific PET Camera. We calibrate the time delay correction factors for each detector module in the camera using two methods-using the Time Alignment Probe (which measures the time difference between the probe and each detector module) and using the conventional method (which measures the timing difference between all module-module combinations in the camera). These correction factors, which are quantized in $\mathbf{2} \mathbf{n s}$ steps, are compared on a module-by-module basis. The values are in excellent agreement-of the 80 correction factors, 62 agree exactly, 17 differ by 1 step, and 1 differs by 2 steps. We also measure on-time and off-time counting rates when the two sets of calibration factors are loaded into the camera and find that they agree within statistical error. We conclude that the performance using the Time Alignment Probe and conventional methods are equivalent.
\end{abstract}

Index Terms-calibration, positron emission tomography (PET), timing.

\section{INTRODUCTION}

Coincidence time alignment, in which the (fixed) module to module variations in propagation time are corrected for, is an important part of the calibration of a PET camera. This calibration is usually performed using a positron source placed between the two detector modules in question, histogramming the difference in arrival times, and adjusting the time delay of one module so that the distribution is centered at zero time difference. By placing the source between all possible pairs of detectors modules (which is easily done using an orbiting transmission source or a moderately large phantom), the individual delays for each module can be obtained.

Recently, a Time Alignment Probe was developed by Scanwell Systems, Montreal, Canada [1, 2]. It consists of a positron-emitting source embedded in plastic scintillator that is coupled to a photomultiplier tube. Coincidences are generated when the emitted positron excites the plastic scintillator and one of the annihilation photons excites a detector module in the PET camera a brief time later. Positioning of the probe is not critical-it can be placed

Manuscript received Dec 1, 2005. This work was supported in part by the U.S. Department of Energy under contract No. DE-AC02-76CH03000, and in part by Public Health Service Grant No. R33-EB001928.

W. W. Moses is with Lawrence Berkeley National Laboratory, Berkeley, CA 94720 USA (telephone: ++1-510-486-4432, e-mail: wwmoses@lbl.gov).

C. J. Thompson is with Montreal Neurological Institute, McGill University, Montreal, Canada (e-mail: christopher.thompson@mcgill.ca). anywhere that it can be "seen" by the detector module being calibrated.

One advantage of this device is that it simplifies the calibration algorithm. With conventional timing algorithms, time differences between two modules are measured, and this time difference is calibrated out either by adding a delay to one of the modules or removing delay from the other. As each module is placed in coincidence with many other modules in the camera, there is considerable coupling between the individual delay settings. While this is a surmountable problem, it adds complexity to the calibration algorithm. In contrast, the Time Alignment Probe can always be used as the reference detector-its delay is held fixed throughout the calibration procedure and the delays of each of the detector modules are trivially adjusted, as there is no coupling between modules.

While the Time Alignment Probe has previously been described and characterized [2], it has not previously been used to calibrate a PET camera. The purpose of this paper is to perform such a calibration and compare the results to calibration performed with the conventional method.

\section{MEthoDS}

\section{A. Time Alignment Probe}

The Scanwell Time Alignment Probe consists of a $10 \mu \mathrm{Ci}$ ${ }^{22} \mathrm{Na}$ source embedded in polyvinyl toluene-based ASI-200 plastic scintillator (Alpha Spectra Inc., Grand Junction, CO), which is coupled to a Hamamatsu R-1635 photomultiplier

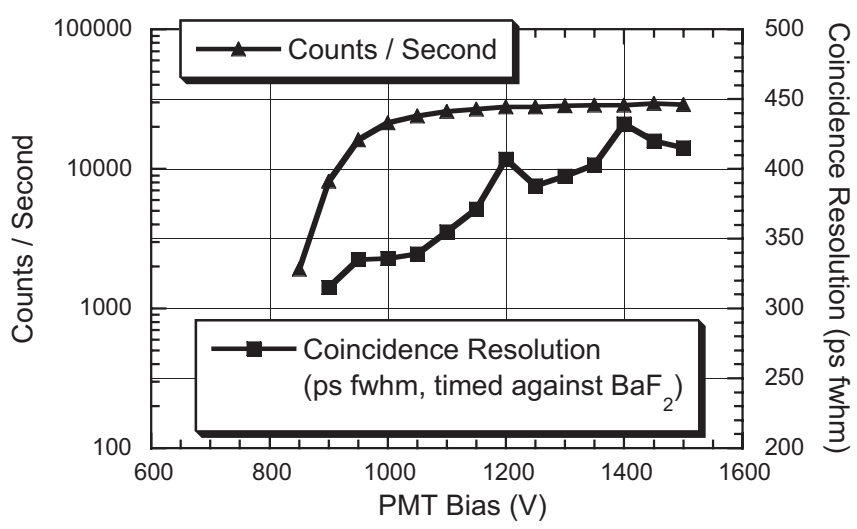

Figure 1: Timing Resolution and Count Rate of the Timing Alignment Probe. This figure shows the coincidence timing resolution and the counting rate of the Timing Alignment Probe as a function of the PMT bias voltage. At the nominal operating voltage of $1250 \mathrm{~V}$, the coincidence timing resolution is approximately $400 \mathrm{ps}$ fwhm. 
tube. The positron ranges out in the plastic scintillator, generating a signal in the PMT. The positron annihilates to form a pair of $511 \mathrm{keV}$ gamma rays, either one of which can then excite a detector module in the PET camera. Note that the coincidences used for calibration are between the emitted positron and one of the annihilation photons, as opposed to the pair of annihilation photons.

Before the Time Alignment Probe is used, we measure its performance as a function of photomultiplier tube bias voltage. We measure the intrinsic timing resolution by putting the Probe in coincidence with a $1 \mathrm{~cm}$ cube of $\mathrm{BaF}_{2}$ scintillator coupled to a Hamamatsu H5321 photomultiplier tube. The Time Alignment Probe and the scintillator / PMT combination are read out with separate channels of a Canberra 454 constant fraction discriminator (CFD), and the time difference between the two signals measured with an Ortec 556 time to digital converter (TAC) digitized by a 16bit analog to digital converter (ADC). The measured coincidence timing resolution, which is shown in Figure 1, has a timing resolution of $400 \mathrm{ps}$ fwhm at the nominal operating voltage of $1250 \mathrm{~V}$. As the $\mathrm{BaF}_{2}$ scintillator / PMT combination has a timing accuracy of $150 \mathrm{ps}$ fwhm, the resolution of the Time Alignment Probe is $370 \mathrm{ps}$ fwhm. This is consistent with the $320 \mathrm{ps}$ fwhm time resolution listed in the manufacturer's catalog for a quartz-windowed version of this photomultiplier tube coupled to $\mathrm{BaF}_{2}$ scintillator [3]. While the timing resolution of the Probe could probably be improved using a higher performance photomultiplier tube, it is more than adequate for all existing PET cameras. Figure 1 also shows the coincident counting rate for this coincidence timing resolution measurement as a function of bias voltage. It shows that the counting rate for the probe is insensitive to bias voltage as long as the bias voltage is above $1 \mathrm{kV}$.

\section{B. Prostate-Specific PET Camera}

One factor that makes the Time Alignment Probe awkward to use is that most PET cameras do not have any spare analog input channels. Therefore, it is virtually impossible to put the Time Alignment Probe into coincidence with any of the detector modules in the PET camera without removing one of the detector modules and plugging the Probe into its electronics. The LBNL Prostate-Specific PET Camera [4-6], however, has 10 unused electronics channels distributed around the tomograph ring. In addition, this camera does not have an orbiting positron source for attenuation correction, and so conventional methods for time calibration are more difficult. We therefore investigate calibrating this camera using the Scanwell Time Alignment Probe.

The detector modules in this PET camera are identical to those used in the CTI HR+ PET camera [7, 8]. They are block detector modules, with an $8 \times 8$ array of $30 \mathrm{~mm}$ deep BGO crystals that cover a $50 \mathrm{~mm} \times 50 \mathrm{~mm}$ area. The electronics are a modified version of the electronics used for the CTI HRRT camera [9-13], with the main difference being that the ASIC used for processing the detector module signal is designed for BGO-based block detectors [14] rather than LSO-based block detectors [15-17]. For this work, the

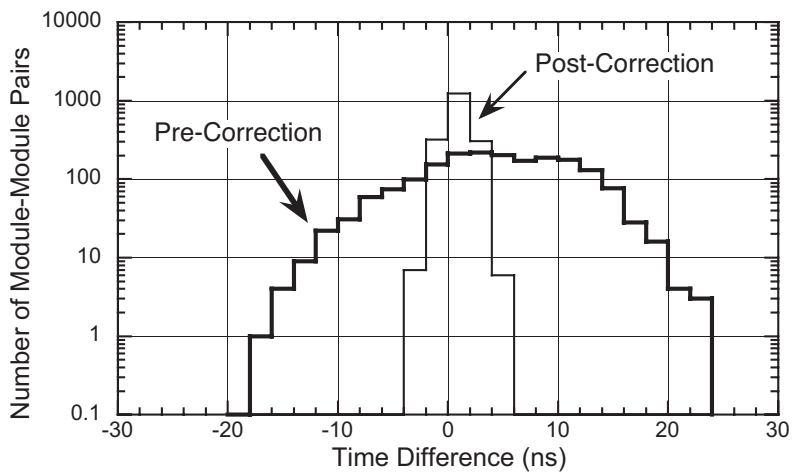

Figure 2: Distribution of Time Differences. This figure shows the distribution of time differences between each valid module-module pair in the camera. Data is shown both pre-correction (all timing delays set to zero) and post-correction (delays set to the values determined by the timing calibration algorithm). The narrow post-correction distribution indicates that the timing calibration is effective.

pertinent features of these electronics are that the arrival time of each annihilation photon is quantized in $2 \mathrm{~ns}$ steps and that the electronics supports an additive time delay correction factor for each crystal that is also quantized in $2 \mathrm{~ns}$ steps. However, we have determined that the crystal-to-crystal time delay variations within a module are small, and so all the delay correction factors within a module are set to the same value, effectively yielding module-dependent delay correction factors.

When operated at $1250 \mathrm{~V}$, the amplitude of the analog signal out of the Time Alignment Probe is well-matched to the analog electronics in the Prostate Camera. The camera's analog input channels are designed for a block detector module and so have inputs for four PMTs, but the Time Alignment Probe is plugged into the input for one of the PMTs. The channel must be calibrated (amplifier gain and discriminator threshold), but the appropriate settings are virtually identical to those used for the block detector. Similarly, the energy threshold parameter must be set, but this too is trivially obtained and similar to that used with BGO block detectors.

\section{MEASUREMENTS}

\section{A. Pre-Calibration Performance}

Before calibrating the Prostate Camera, we set all the delay correction factors to zero, place a $20 \mathrm{~cm}$ diameter positronemitting phantom in the field of view of the camera, and acquire coincident data in a special calibration mode where we record the difference in arrival time of the two detections forming the coincidence. The arrival time for each event is digitized with a 2 ns least count. From these data we generate the time difference distribution for each module-module pair and fit it with a Gaussian function from which we extract the mean (i.e., the average timing difference for each modulemodule pair) and the standard deviation (i.e., the timing resolution for this module-module pair). We histogram this mean timing difference for each module-module pair in Figure 2. The broad distribution, with mean differences as 
Submitted to IEEE Transactions on Nuclear Science

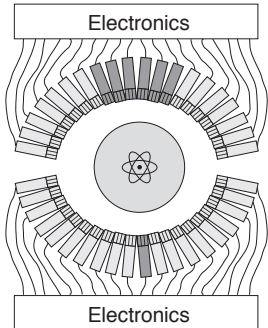

a)

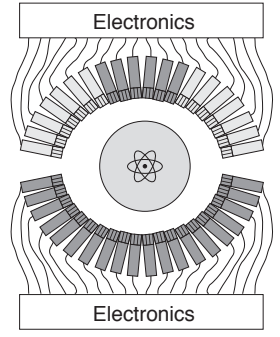

b)

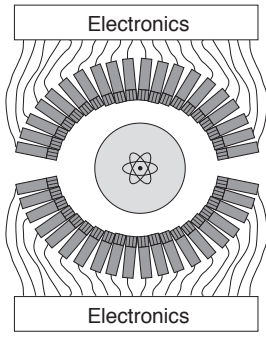

c)
Figure 3: Conventional Calibration Method. a) A single module in the lower arc is used as the reference, and delays for the central modules in the upper arc are adjusted. b) The central modules in the upper arc are used as the reference, and delays for the modules in the lower arc are adjusted. c) The modules in the lower arc are used as the reference, and delays for the modules in the upper arc are adjusted.

large as $22 \mathrm{~ns}$, indicates that the camera badly needs time calibration.

\section{B. Conventional Time-Delay Calibration Method}

We use data similar to that collected in Section III.A. to calibrate the camera using the "conventional" method. A set of fixed delays (one for each module) is computed, with the delay selected in order to place the mean timing difference for each module-module pair as close to zero as possible. As the correction factors are coupled (a time difference between two modules can be eliminated by adding a delay to one module, subtracting this delay from the other module, or applying a partial correction to both modules), we describe the algorithm used to obtain the delays below.

A uniform $20 \mathrm{~cm}$ diameter positron-emitting phantom is placed near the center of the field of view of the camera and all delays are initially set to zero. One detector module in the center of the lower arc is arbitrarily chosen as the "reference" detector module. The time delays between this reference module and a portion of the detector modules in the upper arc are obtained (Figure 3a). These time differences are considered to be the calibration factors for the central modules in the upper arc and are loaded into the electronics for these modules. Only the central portion is calibrated at this point because the phantom is not large enough to excite valid coincidences between the reference module and all of the modules in the upper arc.

The phantom is left in the same position and more data acquired. This time the central detector modules in the upper arc are used as the reference detectors, and the timing delays of all of the modules in the lower arc are adjusted to bring them into alignment with this group of reference detectors (Figure 3b). These delay factors are loaded into the electronics for the modules in the lower arc and a third set of data accumulated. For this final set of data, the modules in the lower arc are used as the reference detectors and the delay factors for all of the modules in the upper arc are obtained and loaded into the electronics (Figure 3c).

The time delay factors for all 80 detector modules are recorded, loaded into the electronics, and time difference data similar to that collected in Section III.A. is obtained. A postcalibration analysis of the module-module time differences

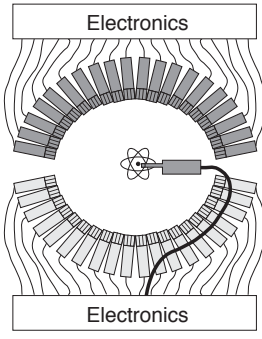

a)

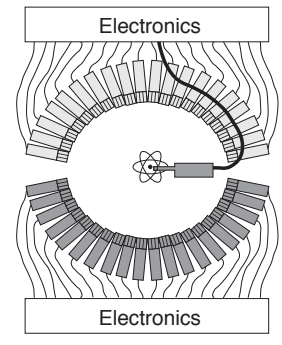

b)
LBNL-59117

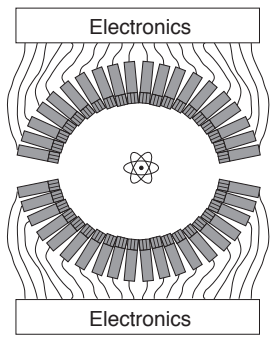

c)
Figure 4: Probe Calibration Method. a) The Timing Probe is used as the reference, and delays for the modules in the upper arc are adjusted. b) The Timing Probe is used as the reference, and delays for the modules in the lower arc are adjusted. c) The relative shift between the upper arc and lower is removed using coincidences between the two arcs.

(identical to that described in Section III.A.) is performed, and the resulting distribution is also plotted in Figure 2. This distribution is considerably narrower than the pre-correction distribution, with most pairs showing 0 or 2 ns mean time differences, demonstrating that the calibration method is effective.

\section{Time Calibration Probe Method}

We then reset all delays to zero and re-calibrate the delays in the camera using the Scanwell Time Alignment Probe. To do this, the probe is placed near the center of the camera and plugged into a spare electronics channel that is located near the center of the lower arc. Time difference data between the probe and all of the modules in the upper arc are acquired (Figure 4a) and used to obtain the delay correction factors for the modules in the upper arc, and these factors loaded into the electronics. As the electronics does not support coincidences between detector modules that are near each other, none of the modules in the lower arc can be calibrated at this point. Therefore, the probe is plugged into a spare electronics channel located near the center of the upper arc (Figure 4b), more data collected, and delay correction factors for the lower arc are obtained and loaded into the electronics. All the modules in the upper arc are now aligned to each other and all the modules in the lower arc are aligned to each other, but an overall shift between the upper and lower arc is possible. To remove this shift, coincident events between modules in the upper arc and modules in the lower arc are obtained (Figure 4c), a single additive factor that corrects for this shift is obtained, and this additional factor is added to all of the factors in one of the arcs. The time delay factors for all 80 detector modules (obtained with the Probe) are recorded and loaded into the electronics.

\section{Comparison of Delay Calibration Factors}

To compare the two calibration methods we first examine, module by module, the delay calibration factors for all 80 modules that were obtained by the conventional and Probe methods. Of the 80 delays, 62 agree exactly, 17 differ by 1 step (or $2 \mathrm{~ns}$ ), and 1 differs by 2 steps (or $4 \mathrm{~ns}$ ). This indicates that the Scanwell probe and the conventional method yield virtually identical calibration values. The differences are consistent with quantization error and would appear to be 
Table 1: On Time, Off Time, and On Time minus Off Time event rates (in counts per second) when the Prostate PET camera uses module-dependent delay calibration factors obtained using the Conventional method and with the Time Alignment Probe. The difference in the rates obtained with both methods is also shown. The errors shown are the statistical error.

\begin{tabular}{|c|c|c|c|c|c|c|c|c|c|}
\hline \multirow[t]{2}{*}{$\begin{array}{l}\text { Window } \\
\text { Width }\end{array}$} & \multicolumn{3}{|c|}{ Conventional Method } & \multicolumn{3}{|c|}{ Probe } & \multicolumn{3}{|c|}{$\begin{array}{c}\text { Difference } \\
\text { (Conventional Method - Probe) }\end{array}$} \\
\hline & $\begin{array}{l}\text { On Time } \\
\text { (cts/sec) }\end{array}$ & $\begin{array}{l}\text { Off Time } \\
\text { (cts/sec) }\end{array}$ & $\begin{array}{l}\text { On-Off } \\
\text { (cts/sec) }\end{array}$ & $\begin{array}{l}\text { On Time } \\
\text { (cts/sec) }\end{array}$ & $\begin{array}{l}\text { Off Time } \\
\text { (cts/sec) }\end{array}$ & $\begin{array}{l}\text { On-Off } \\
\text { (cts/sec) }\end{array}$ & $\begin{array}{l}\text { On Time } \\
\text { (cts/sec) }\end{array}$ & $\begin{array}{l}\text { Off Time } \\
\text { (cts/sec) }\end{array}$ & $\begin{array}{l}\text { On-Off } \\
\text { (cts/sec) }\end{array}$ \\
\hline $2 \mathrm{~ns}$ & $15688 \pm 47$ & $1741 \pm 16$ & $13947 \pm 50$ & $15842 \pm 48$ & $1760 \pm 16$ & $14082 \pm 50$ & $-154 \pm 67$ & $-19 \pm 22$ & $-135 \pm 71$ \\
\hline $6 \mathrm{~ns}$ & $40037 \pm 76$ & $5301 \pm 28$ & $34736 \pm 80$ & $39873 \pm 75$ & $5180 \pm 27$ & $34693 \pm 80$ & $164 \pm 107$ & $121 \pm 39$ & $43 \pm 114$ \\
\hline $10 \mathrm{~ns}$ & $51787 \pm 86$ & $8624 \pm 35$ & $43163 \pm 93$ & $51862 \pm 86$ & $8657 \pm 35$ & $43205 \pm 93$ & $-76 \pm 122$ & $-33 \pm 50$ & $-43 \pm 131$ \\
\hline $14 \mathrm{~ns}$ & $57773 \pm 91$ & $12005 \pm 41$ & $45768 \pm 100$ & $57913 \pm 91$ & $12007 \pm 41$ & $45906 \pm 100$ & $-140 \pm 129$ & $-2 \pm 59$ & $-138 \pm 141$ \\
\hline $18 \mathrm{~ns}$ & $62227 \pm 94$ & $15525 \pm 47$ & $16701 \pm 105$ & $62045 \pm 94$ & $15519 \pm 47$ & $46525 \pm 105$ & $182 \pm 133$ & $6 \pm 67$ & $176 \pm 149$ \\
\hline $22 \mathrm{~ns}$ & $65453 \pm 97$ & $18770 \pm 52$ & $46683 \pm 110$ & $65809 \pm 97$ & $18816 \pm 52$ & $46993 \pm 110$ & $-355 \pm 137$ & $-45 \pm 73$ & $-310 \pm 155$ \\
\hline
\end{tabular}

irrelevant, as this BGO-based camera has 6-8 ns fwhm coincidence timing resolution and uses a $10 \mathrm{~ns}$ wide coincidence window.

\section{E. Comparison of Performance}

Although the results described in Section III.D. indicate that the delay calibration factors obtained using the two calibration methods are nearly identical, they are slightly different, and this begs the question whether one of the methods out-performs the other. We investigate this question by assuming that the more accurate calibration method would give a higher Trues to Randoms ratio than the less accurate method, with the improvement depending on the coincidence window width [18]. The underlying assumption is that when narrow coincidence windows are used, some of the true coincidence events that are accepted by a well-calibrated system will be missed when the system is poorly calibrated. The random event rate, on the other hand, depends only on the window width and not the quality of the calibration factors. Therefore, the largest improvement should be seen at the narrowest coincidence window width and minimal improvement should be seen at wide coincidence window widths.

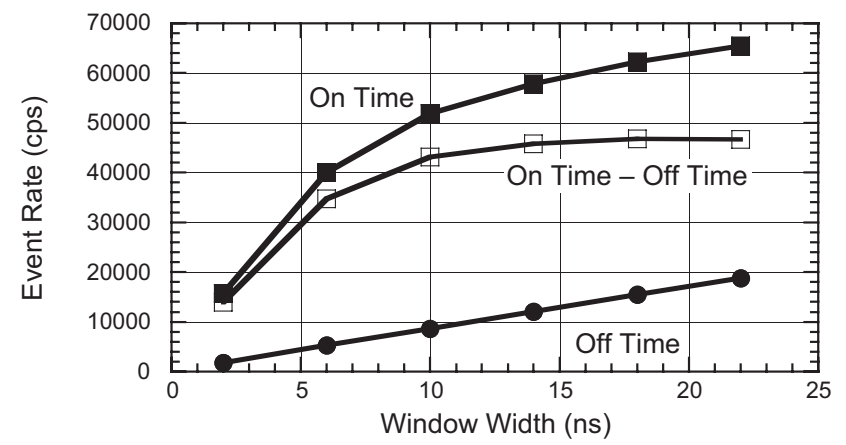

Figure 5: On Time, Off Time (Randoms), and On Time minus Off Time (Trues + Scatter) rates as a function of coincidence window width.
We place a $20 \mathrm{~cm}$ diameter, $20 \mathrm{~cm}$ long phantom filled with $557 \mu \mathrm{Ci}$ of ${ }^{68} \mathrm{Ge}$ in the center of the camera, measure the on-time and off-time event rates obtained under various datataking conditions (different coincidence window widths and different sets of the calibration factors), and compute the Trues+Scatter (On Time minus Off Time) and Random (Off Time) rates for each data-taking condition. A small correction $(0.5 \%)$ is applied to one data set to correct for radioactive decay of the source. These rates are plotted in Figure 5 and are shown in Table 1. As expected, the Randoms event rate is proportional to the coincidence window width. The dependence of the Trues + Scatter event rate on coincidence window width is also as expected-the rate is nearly independent of width for values $\geq 14 \mathrm{~ns}$ (which is wide enough that essentially all of the prompt events are accepted) but drops significantly as the width decreases below this value. Only the data taken with the conventional calibration method is shown in Figure 5, as the data taken with both methods is effectively identical.

Table 1 shows the event rates and their statistical errors in more detail. These data show that for all coincidence window widths, all of the event rates agree within statistical errors, and so are independent of the calibration method used to obtain the delay values. We therefore conclude that for this PET camera, neither method (conventional or Probe) outperforms the other.

\section{DISCUSSION}

Given that the Probe and the conventional method produce equally good delay time calibration values, what other factors are important in the choice of which method to use? For this prostate camera (which does not have an orbiting positron source), the main drawback of the conventional method is that it requires a positron source be placed in the field of view of the camera such that it excites all module-module pairs. As 
our phantom does not cover the entire field of view, we must either take several data acquisitions or manually move a source throughout the field of view. In addition, the calibration algorithm is complicated by the fact that the delay values are coupled (as described in Section III.B.). However, these problems are easily surmounted.

The main drawback of the Scanwell Probe is that the electronics for virtually all existing PET cameras are configured such that none of the electronics channels is in coincidence with all of the other channels. Thus, there is no single channel that we can plug the calibrator into and calibrate the entire camera, and so we must take two data sets (with the Time Alignment Probe plugged in to two separate channels) and then reconcile them. In addition, the source strength for the Probe is limited by dead time considerations and so may not provide as high a count rate (and thus a longer data acquisition time) as the conventional method. Although these features cause some practical problems with existing cameras, neither is a fundamental issue. These problems could be easily solved by adding a dedicated electronics channel that has significantly less dead time than the electronics in the prostate camera (which has a dead time appropriate for $\mathrm{BGO}$, not plastic scintillator) and is in coincidence with all of the detector modules in the camera.

Although these data were acquired from a single PET camera, we believe that the measurements and conclusions should be valid for most commercial, BGO-based PET cameras, as the design and performance (detector modules, alectronics, time digitization accuracy, and timing resolution) of these cameras are very similar. Whole-body PET cameras that have better timing performance, such as LSO-based systems $[9,10,12,13]$, have finer time digitization accuracy and better timing resolution and so there is no guarantee that these results will be valid for those systems. However, the intrinsic timing accuracy of the Probe is high (370 ps fwhm) and can be improved with a higher performance photomultiplier tube, and so we expect that these results will be valid for LSO-based PET cameras.

\section{CONCLUSIONS}

We have used the Scanwell Time Alignment Probe to calibrate the time delay values for the BGO-based LBNL prostate-specific PET camera. We find that the Probe is easy to use, performs as expected, and provides calibration factors that are effectively identical to those obtained with the conventional method. While the conventional and Probe calibration methods have some relative advantages and disadvantages, none are either large or fundamental. Thus, the choice of which method and hardware to use is largely a matter of personal preference.

\section{ACKNOWLEDGMENT}

We thank Dr. G.C. Wang of GE Global Research and Drs. J.S. Huber and W.-S. Choong of LBNL for their help in developing the prostate camera. This work was supported in part by the Director, Office of Science, Office of Biological and Environmental Research, Medical Science Division of the U.S. Department of Energy under Contract No. DEAC03-76SF00098, and in part by the National Institutes of Health, National Institute of Biomedical Imaging and Bioengineering under grant No. R33-EB001928. Reference to a company or product name does not imply approval or recommendation by the University of California or the U.S. Department of Energy to the exclusion of others that may be suitable.

\section{REFERENCES}

[1]http://www.scanwell.ca/

[2]C. J. Thompson, M.-L. Camborde, and M. E. Casey, "A central positron source to perform the timing alignment of detectors in a PET scanner," IEEE Trans. Nucl. Sci., vol. NS-52, (accepted for publication), Oct. 2004.

[3] "Data Sheet for the R2496 Photomultiplier Tube," Hamamatsu Photonics Data Sheet No. TPMH1229E01, pp. 1-2, Sept. 1998.

[4]J. S. Huber, S. E. Derenzo, J. Qi, W. W. Moses, R. H. Huesman, et al., "Conceptual design of a compact positron tomograph for prostate imaging," IEEE Trans. Nucl. Sci., vol. NS-48, pp. 1506-1511, Aug. 2001.

[5]J. Qi, J. S. Huber, R. H. Huesman, W. W. Moses, S. E. Derenzo, et al., "Septa design for a prostate specific PET camera," IEEE Trans. Nucl. Sci., vol. NS-52, pp. 107-113, Feb. 2005.

[6] J. S. Huber, W. S. Choong, W. W. Moses, J. Qi, J. Hu, et al., "Characterization of a PET camera optimized for prostate imaging," IEEE Trans. Nucl. Sci., vol. NS-53, (submitted for publication), 2006.

[7]L.-E. Adam, J. Zaers, H. Ostertag, H. Trojan, M. E. Bellemann, et al., "Performance evaluation of the whole-body PET scanner ECAT EXACT HR+ following the IEC standard," IEEE Trans. Nucl. Sci., vol. NS-44, pp. 1172-1179, June 1997.

[8]T. Hasegawa, Y. Wada, H. Murayama and T. Nakajima, "Basic performance of the PET scanner, EXACT HR+, with adjustable dataacquisition parameters," IEEE Trans. Nucl. Sci., vol. NS-46, pp. 652658 , June 1999.

[9] W. F. Jones, J. H. Reed, J. L. Everman, J. W. Young and R. D. Seese, "Next generation PET data acquisition architectures," IEEE Trans. Nucl. Sci., vol. NS-44, pp. 1202-1207, June 1997.

[10] J. W. Young, J. C. Moyers, and M. Lenox, "FPGA based front end electronics for a high resolution PET scanner," IEEE Trans. Nucl. Sci., vol. NS-47, pp. 1676-1680, Aug. 2000.

[11] W. W. Moses, J. W. Young, K. Baker, W. Jones, M. Lenox, et al., "The electronics system for the LBNL positron emission mammography (PEM) camera," IEEE Trans. Nucl. Sci., vol. NS-48, pp. 632-636, June 2001.

[12] M. Schmand, L. Eriksson, M. E. Casey, M. S. Andreaco, C. Melcher, et al., "Performance results of a new DOI detector block for a High Resolution PET-LSO Research Tomograph HRRT," IEEE Trans. Nucl. Sci., vol. NS-45, pp. 3000-3006, Dec. 1998.

[13] K. Wienhard, M. Schmand, M. E. Casey, K. Baker, J. Bao, et al., "The ECAT HRRT: performance and first clinical application of the new high resolution research tomograph," IEEE Trans. Nucl. Sci., vol. NS49, pp. 104-110, Feb. 2002.

[14] D. M. Binkley, "Performance of non-delay-line constant-fraction discriminator timing circuits," IEEE Trans. Nucl. Sci., vol. 41, pp. 1169-1175, Aug. 1994.

[15] D. M. Binkley, B. S. Puckett, B. K. Swann, J. A. Rochelle, M. S. Musrock, et al., "A $10-\mathrm{mc} / \mathrm{s}, 0.5-\mu \mathrm{m}$ CMOS constant-fraction discriminator having built-in pulse tail cancellation," IEEE Trans. Nucl. Sci., vol. NS-49, pp. 1130-1140, June 2002.

[16] B. K. Swann, J. M. Rochelle, D. M. Binkley, B. S. Puckett, B. J. Blalock, et al., "A custom mixed-signal CMOS integrated circuit for high performance PET tomograph front-end applications," IEEE Trans. Nucl. Sci, vol. NS-50, pp. 909-914, Aug. 2003.

[17] M. S. Musrock, J. W. Young, J. C. Moyers, J. E. Breeding, M. E. Casey, et al., "Performance characteristics of a new generation of processing circuits for PET applications," IEEE Trans. Nucl. Sci., vol. NS-50, pp. 974-978, Aug. 2003. 
[18] C. J. Thompson and A. L. Goertzen, "A method for determination of the timing stability of PET scanners," IEEE Trans. Med. Img., vol. 24, pp. 1053-1057, Aug. 2005. 